$\begin{array}{ll}\text { Research Square } & \begin{array}{l}\text { Preprints are preliminary reports that have not undergone peer review. } \\ \text { They should not be considered conclusive, used to inform clinical practice, } \\ \text { or referenced by the media as validated information. }\end{array}\end{array}$

\title{
The First Case of Leucopenia Campylobacter Jejuni Infection in Hepatitis B Cirrhosis with Bacteremia and Peritonitis in Asia
}

\author{
Chai jiatong \\ Jilin University First Hospital \\ Zeyu Sun \\ Jilin University First Hospital \\ Yiting Wang \\ Jilin University First Hospital \\ Chun Yang \\ Jilin University First Hospital \\ Jiancheng Xu ( $\nabla$ xjc@jlu.edu.cn) \\ Jilin University First Hospital https://orcid.org/0000-0001-8796-271X
}

\section{Case report}

Keywords: Campylobacter jejuni, Liver Cirrhosis, Bacteremia, Spontaneous bacterial peritonitis, Leukopenia

Posted Date: September 13th, 2021

DOI: https://doi.org/10.21203/rs.3.rs-868418/v1

License: (c) (1) This work is licensed under a Creative Commons Attribution 4.0 International License. Read Full License 


\section{Abstract}

Backgroud: Bacteremia and peritonitis caused by Campylobacter jejuni are rarely found in hepatitis B cirrhotic patients, and leukopenia rarely occurs in cases of bacterial infection. Hence, more cases should be shared to improve antibiotic treatment strategies and avoid missed diagnosis in clinical practice.

Case presentation: This report shared a case of Campylobacter jejuni infection in hepatitis B cirrhosis with leukopenia that has never been reported in Asia. A 59-year-old with occult hepatitis B cirrhosis was hospitalized due to persistent mild fever and obvious abdominal distention. The patient was diagnosed with bacteremia and spontaneous bacterial peritonitis, and was discharged after treatment with Cefminox Sodium and CefepiMe Hydrochlorid.

Conclusion: Parenteral infection with Campylobacter jejuni is more common in patients with decreased immune function. Bacterial infection concealed by leucopenia should be considered to avoid delaying treatment in patients with low immune function.

\section{Background}

Campylobacter jejuni (C. jejuni) represents one of the most common identified bacterial pathogens causing acute gastroenteritis in worldwide[1]. It usually colonizes the lower gastrointestinal tract and can cause mild and self-limited gastroenteritis that are characterized by acute diarrhea with potential blood in stools, fever, and abdominal pain[2]. However, reports on Campylobacterinfections, are rarely founded in extra-intestinal infections, especially with bacteremia and peritonitis. The risk of developing a severe infection from $C$. jejuni would increase in patients with immunosuppression (i.e., those with immunodeficiency, HIV, diabetes, cirrhosis, or cancer, and those undergoing chemotherapy). Hence, the significance of clinical suspicions of Campylobacter spp. and accurate use of antibiotics in the above patients should be emphasized, which is due to the slow growth rate of pathogens and the rapid emergence of antimicrobial agentresistant Campylobacter strains all over the world[3,4]. Liver cirrhosis caused by various hepatitis can lead to the decline of human immune function, which may provide an opportunity for bacterial invasion. Reports of alcoholic and hepatitis $C$ cirrhosis caused by $C$. jejuni infection have been found in Asia, but no cases of $C$. jejuni infection caused by hepatitis B cirrhosis have been reported. Besides, leukopenia usually occurs in patients with decreased immune function, but it is rarely found in cases of bacterial infection, which would lead to missed diagnosis of bacterial infection and followed by the delays in treatment. Report of new infection cases with leukopenia would help consummate the treatment strategy of $C$. jejuni infection and provide reference and warning for clinical practice not only in Asia but also other western countries. In this report, we reported a case of a female patient with decompensated hepatitis B cirrhosis and leukopenia who suffered from $C$. jejuni bacteremia and peritonitis, which was different with common cases.

\section{Case Presentation}

A 59-year-old woman was hospitalized due to a 10-days history of abdominal bloating, fever, edema of lower limbs and postprandial vomit. She had a history of Hepatitis B, and developed to the decompensated period with cirrhosis 3 years ago. The patient had a history of taking entecavir for 3 years and stopped taking the medicine half a year ago. The patient denied the history of other diseases or food and drug allergy.

Her initial vital signs were: blood pressure, $95 / 52 \mathrm{mmHg}$; heart rate, 96 beats $/ \mathrm{min}$; respiratory rate, 18 breaths $/ \mathrm{min}$; body temperature, $36.5^{\circ} \mathrm{C}$ and with ascites. Physical examination was remarkable for scoliosis, icteric sclera, abdominal distension and positive abdominal fluid shift. On admission, a chest radiography showed scattered inflammation in both lungs. The laboratory results are shown in Table 1. Blood and ascites culture were performed for further evaluation. BACT/ALERT FN Plus Aerobic/F was chosen as containers for culturing bacteria in automatic blood culture system (BACT/ALERT VIRTUO) under aerobic conditions, the process of culture was shown in Fig. 1.

Table 1 Labrotaty results

\begin{tabular}{|lll|}
\hline Laboratory analytes & Results & Reference range \\
\hline Leukocyte $\left(\times 10^{9} / \mathrm{L}\right)$ & 2.83 & $3.5-9.5$ \\
\hline Ascites leukocyte $\left(\times 10^{6} / \mathrm{L}\right)$ & 71 & - \\
\hline Haemoglobin $(\mathrm{g} / \mathrm{L})$ & 86 & $115-150$ \\
\hline High sensitivity C-reactive protein $(\mathrm{mg} / \mathrm{L})$ & 36.36 & $0-3.5$ \\
\hline Aspartate aminotransaminase $(\mathrm{U} / \mathrm{L})$ & 57 & $13-35$ \\
\hline Glutamine transaminase $(\mathrm{U} / \mathrm{L})$ & 48.2 & $7.0-45$ \\
\hline Alanine aminotransaminase $(\mathrm{U} / \mathrm{L})$ & 24.1 & $7.0-40$ \\
\hline Cholinesterase $(\mathrm{U} / \mathrm{L})$ & 1492 & $3930-10800$ \\
\hline Alkaline phosphatase $(\mathrm{U} / \mathrm{L})$ & 119.9 & $50-135$ \\
\hline Albumin $(\mathrm{g} / \mathrm{L})$ & 22.6 & $40-55$ \\
\hline Total bilirubin (umol/L) & 89.5 & $0-21.0$ \\
\hline
\end{tabular}

On the second day of hospitalization, a fever of $37.8^{\circ} \mathrm{C}$ was recorded, and Cefminox Sodium $(0.25 \mathrm{~g})$ was administered as an empirical antibiotic to treat abdominal infection for sixth days, but the patient was still with a fever and massive ascites after antimicrobial therapy. On the fifth day of hospitalization, her bloodstream and ascites cultures were positive in Columbia agar. After inoculation on the first day, the pathogen was transferred and continue to be cultured. 
The culture medium was placed in a micro oxygen environment and cultured at $37^{\circ} \mathrm{C}$ for 2 days after the culture transformation. On the third day of hospitalization, the alarm of the automatic culture system indicated that the blood culture was positive, and the clinician then added voriconazole (50mg) to the patient. The strain was separated and found with spiral and active status under microscope on the fifth day of hospitalization, which led to the the suspicion of $C$. jejuni. MALDI-TOF MS (BioMérieux, VITEK MS mass spectrometer) was finally used to identify the strain as $C$. jejuni with $99.9 \%$ probability, the MS results were shown in Fig. 2. Automatic identification and 16S rRNA gen were not performed due to the deficiency of bacterial quantity. The biochemical test results showed that the contact enzyme test, nitrite reduction test were positive; nitrite reduction test, hydrogen sulfide test and urease test were negative.

The laboratory test on the sixth day showed that abdominal infection still existed with the leukocyte count of $287 \times 10^{6} / \mathrm{L}$ in ascites. However, the count of leukocyte always showed a lower status than the normal range except for the seventh day of hospitalization. The changes of temperature and partial laboratory test results during hospitalization are shown in Fig. 3. Although the patient always had slight fever, the temperature always maintained a downward trend. Hence, the antibiotics were not replaced even after the blood culture identification result was confirmed as $C$. jejuni. The patient was found to suffer from hypokalemia, hyponatremia and hypocalcemia $\left(\mathrm{K}^{+}, 3.27 \mathrm{mmol} / \mathrm{L} ; \mathrm{Na}^{+}, 135.6 \mathrm{mmol} / \mathrm{L} ; \mathrm{Ca}^{2+}, 1.81 \mathrm{mmol} / \mathrm{L}\right)$ and treated with potassium supplements (Potassium citrate: $2.9 \mathrm{~g}$ ). The patient was relived of fever on the seventh day of hospitaliztion, but with a recurrent fever on the next day. The antibiotic was changed to CefepiMe Hydrochlorid ( $2 \mathrm{~g} /$ day) which was continued for five days, and she responded well to this treatment regimen. Blood and ascites culture were performed again to check bacterial retention, and showed negative results. Ascites drainage decreased gradually and abdominal pressure returned to normal in the last two days of hospitalization, and discharge approval was given after clinical evaluation. The patient had no diarrhea symptoms throughout hospitalization. Unfortunately, no colonies were cultured for drug sensitivity test due to the difficult culture of $C$. jejuni. Our patient had a severe liver disease that led to significant immunosuppression, which might be an important cause of $C$. jejuni infection.

\section{Discussion And Conclusions}

Bacteremia was rarely found in Campylobacteriosis, with $C$. jejuni and $C$. coli found to be the most common causes among the cases[5]. A previous study showed that up to $93 \%$ of the bacteremia cases are related to the recognized risk factors of immune function decline or immunosuppression, such as chronic liver disease, human immunodeficiency virus infection, malignant tumor and humoral immunodeficiency[6]. Though Campylobacter bacteremia might resolve without antibiotic treatment in a normal host[4], appropriate selection of empirical antibiotics from clinicians and definite confirmation from laboratories were of great importance to the outcome and prognosis of immunocompromised patients. In addition, the studies of bacteremia combined with spontaneous bacterial peritonitis (SBP) caused by Campylobacterwere more rarely found in the database. Peritonitis generally occured in patients with ascites, and patients with liver disease were more likely to form ascites, which provided space and conditions for bacterial growth and reproduction. This report presented the case of $C$. jejuni bacteremia and SBP in a patient with liver cirrhosis. She suffered from fever and ascites, but recovered with the administration of empirical antibiotics and supportive care. It was worth noting that the leukocyte count of the patient were lower than the normal value on admission, so if other symptoms such as fever were not obvious, it was easy to ignore the bacterial infection in diagnosis. Besides, the common diarrhea symptoms caused by $C$. jejuni were not found in this patient, which meant that clinicians should be alert to non-diarrhea symptoms of $C$. jejuni infection to avoid delaying treatment.

Rare relevant publications about liver cirrhosis with Campylobacter infection since 2010 were selected using PUBMED, excluding non English article, as shown in Table 2. Among them, 4 cases were Campylobacter infection patients combined with bacteremia and SBP, and the patients all got a good recovery fortunately. A study hypothesised the hepatic immune response fails to inhibit the haematogenous spread of the infection when the bacteria gain access to the portal circulation in immunocompromised patients[7], which would also lead to the formation of ascites. Besides, it was worth noting that diarrhea didn't always appear as a common first symptom in these cases, which was different from the common Campylobacter infection. The special culture environment led to the difficulty in the growth of Campylobacter, hence, the accuracy of the choice for agar should be emphasized. Brain heart infusion agar plates incubated at $25^{\circ} \mathrm{C}$ and $42^{\circ} \mathrm{C}[8]$, Schaedler and Columbia agar used in this report were able to culture the Campylobacter, and $5 \%$ sheep blood agar and chocolate agar were used to subculture the bacteria for further analysis[9]. However, chocolate agar failed to culture the $C$. jejuni in this report, which might due to the culture conditions and operation technology. Other molecular methods, including MALDI-TOF MS and the 16S rRNA gen analysis, were also useful for rapid identification of strains[8]. Due to the lack of bacteria for further molecular analysis, only MALDI-TOF MS was used in this study to provide faster and more sensitive identifying process in strain typing, epidemiological studies, antibiotic resistance, etc[10]. Besides, biomedical tests were also performed for auxiliary identification in this report, but were uncommon in other reports because these tests were more commonly used in testing known results rather than identifying specific bacterial species from unknown positive results. In the process of culturing $C$. jejuni in the laboratory, positive alarm was generally given in 24-48 hours after acterial culture and transformation. At this time, clinicians could take the lead in empirical medication. C. jejuni would show different growth rates according to the temperature difference of the culture environment in 24-72 hours after the blood culture alarm. The culture temperature after bacterial transformation was $42-43^{\circ} \mathrm{C}$ or $37^{\circ} \mathrm{C}$, which was suitable for the growth of $C$. jejuni. Although inappropriate or delayed appropriate antimicrobial treatment does not seem to be associated with the recovery of patient[11], timely blood or body fluid culture would benefit the prognosis of patients and reduce the incidence of sequelae. 
Table 2

Reported Campylobacter infection cases in liver cirrhosis patients since 2010.

\begin{tabular}{|c|c|c|c|c|c|c|c|c|c|}
\hline \multirow[t]{2}{*}{ Reference } & \multirow[t]{2}{*}{ Country } & \multirow{2}{*}{$\begin{array}{l}\text { Clinical } \\
\text { Manifestation }\end{array}$} & \multirow[t]{2}{*}{ Comorbidities } & \multirow[t]{2}{*}{ Diagnosis } & \multirow{2}{*}{$\begin{array}{l}\text { Leukocyte } \\
\left(\times 10^{9} / \mathrm{L}\right)\end{array}$} & \multirow[t]{2}{*}{ Antibiotics Used } & \multicolumn{2}{|c|}{ Antibiotic Susceptibility Test } & \multirow[t]{2}{*}{1} \\
\hline & & & & & & & Sensitive & Resistant & \\
\hline \multirow[t]{2}{*}{ [4] } & \multirow[t]{2}{*}{ Korea } & [Drowsiness] & \multirow{2}{*}{$\begin{array}{l}\text { [Cryptogenic } \\
\text { liver cirrhosis] }\end{array}$} & \multirow[t]{2}{*}{ [Bacteremia] } & \multirow[t]{2}{*}{5.7} & [Cefotaxime] & \multirow[t]{2}{*}{ [Erythromycin] } & [Ciprofloxacin] & I \\
\hline & & [Fever] & & & & [Azithromycin] & & [Tetracycline] & \\
\hline \multirow[t]{6}{*}{ [12] } & \multirow[t]{6}{*}{ USA } & [Positive & \multirow{6}{*}{$\begin{array}{l}\text { [Hepatitis C } \\
\text { cirrhosis] }\end{array}$} & [Bacteremia] & 2.3 & [Vancomycin] & \multirow[t]{6}{*}{ NM } & & I \\
\hline & & fluid shift] & & [SBP] & & [Doxycycline] & & & \\
\hline & & [Erythematous] & & & & [Imipenem] & & & \\
\hline & & [Edematous] & & & & [Cilastatin] & & & \\
\hline & & & & & & [Ciprofloxacin] & & & \\
\hline & & & & & & [Piperacillin/tazobactam] & & & \\
\hline \multirow[t]{3}{*}{ [13] } & \multirow[t]{3}{*}{ USA } & [Ascites fluid] & \multirow{2}{*}{$\begin{array}{l}\text { [Polycythemia } \\
\text { vera with } \\
\text { splenomegaly] }\end{array}$} & [Bacteremia] & \multirow[t]{3}{*}{4.67} & [Ceftriaxone] & \multirow{3}{*}{$\begin{array}{l}\text { [Erythromycin] } \\
\text { [Ciprofloxacin] }\end{array}$} & \multirow[t]{3}{*}{ NM } & \multirow[t]{3}{*}{1} \\
\hline & & [Fever] & & [SBP] & & [Meropenem] & & & \\
\hline & & & $\begin{array}{l}\text { [Alcoholic liver } \\
\text { cirrhosis] }\end{array}$ & & & & & & \\
\hline \multirow[t]{4}{*}{ [14] } & \multirow[t]{4}{*}{ Thaiwan } & [Fever] & \multirow{4}{*}{$\begin{array}{l}\text { [Alcoholic liver } \\
\text { cirrhosis] } \\
\text { [Type 2 } \\
\text { diabetes] }\end{array}$} & [Bacteremia] & \multirow[t]{4}{*}{8.5} & \multirow[t]{4}{*}{ [Ciprofloxacin] } & \multirow[t]{4}{*}{ NM } & & 1 \\
\hline & & [Abdominal & & [SBP] & & & & & \\
\hline & & pain] & & & & & & & \\
\hline & & $\begin{array}{l}\text { [Abdominal } \\
\text { swelling] }\end{array}$ & & & & & & & \\
\hline \multirow[t]{6}{*}{ [9] } & Japan & [Fatigue] & [Alcoholic liver & [Bacteremia] & 13.1 & [Cefotaxime] & [Ampicillin] & [Minocycline] & 1 \\
\hline & & [Abdominal & cirrhosis] & [SBP] & & [Ampicillin] & [Ceftriaxone] & & \\
\hline & & & [Oesophageal & & & & [Erythromycin] & & \\
\hline & & diarrhoea] & & & & & [Ciprofloxacin] & & \\
\hline & & & & & & & [Gentamicin] & & \\
\hline & & & & & & & [Imipenem] & & \\
\hline
\end{tabular}

It was found that cephalosporins were mainly used in patients with Campylobacter infection in liver cirrhosis, while macrolides and quinolones were mainly used in patients with other diseases such as cancer and HIV infection. In fact, the guidelines suggested the third-generation cephalosporin (such as cefotaxime) for empiric treatment of SBP[6]. Hence, cephalosporins were often used as empirical drugs in the treatment of peritonitis in the absence of blood culture results, and the cirrhotica patients with peritonitis and bacteremia caused by Campylobacter treated with this kind of drugs had achieved good outcomes. However, the therapeutic effect of cephalosporins in other cases remained to be studied, and carbapenems (such as imipenem) are generally considered to be a good choice for radical cure of Campylobacter infection[9]. Besides, fluoroquinolones and macrolides are considered as first-line agents for the treatment of Campylobacterinfections. Nevertheless, high resistance to the above antibacterials were also found in many cases, and might vary in different regions[15]. In addition, clinicians should also take note of the situation that the patients who repeatedly infected with Campylobacter were sensitive to one drug at the beginning, but later developed resistance. Hence, the observation to the dynamic changes of antibiotic sensitivity and appropriate combination therapy were of great importance for the improvement of treatment efficiency.

Due to the particularity of the leukocyte level in this report, the reports of Campylobacter bacteremia since 2011 have also been searched for comparison. Reports that did not give leukocyte data were excluded, and the leukocyte counts of the remaining reports were summarized, as shown in Fig. 4 . Four cases had leukopenia among the enrolled reports, but only one of four patients had cirrhosis with bacteremia and peritonitis, like the present case. Patients with leukocyte count less than $1.0 \times 10^{9} / \mathrm{L}$ had serious primary diseases, including acute lymphoblastic leukemia (ALL), chronic non indolent Hodgkin's lymphoma and immunosuppressive therapy due to kidney transplantation, while leucopenia was rarely found in patients with cirrhosis. It is not surprising that leukopenia occurs in patients with acute lymphoblastic leukemia and Hodgkin's lymphoma due to decreased hematopoietic system function and bone marrow suppression. However, leukopenia was rarely found in patients with cirrhosis. Surprisingly, the leukocyte level in this report showed similar trend with the case of hepatitis $\mathrm{C}$ cirrhosis with $C$. jejuni infection. Declination of immune function might be the leading reason to this phenomenon even in bacterial infection. However, other patients with cirrhosis showed the opposite trend of leukocyte level, which illustrated that leukopenia was not a common feature of bacterial infection in patients with impaired immune function. Even so, the above-mentioned reports warned that the bacterial infection concealed by leukopenia should be considered as a special character in the clinical treatment of immunocompromised patients, so as to avoid the adverse consequences caused by the aggravation of infection.

In conclusion, this is the first report of $C$. jejuni bacteremia and SBP characterized by leucopenia in a patient with hepatitis B cirrhosis in Asia. Careful consideration of bacterial infection and corresponding laboratory examination are needed when the patients with leukopenia and immunosuppression have 
fever and other suspected infection symptoms. The use of antibiotics should be carefully considered for patients with peritonitis and bacteremia, and the combination of drugs can be used when necessary.

\section{Declarations}

\section{Ethics approval and consent to participate}

Not applicable

\section{Consent for publication}

All authors agreed to publish the manuscript

\section{Availability of data and materials}

Not applicable

\section{Competing interests}

There are no conflicts of interest or disclosures to report for any of the authors.

\section{Funding}

This work was supported by First Hospital Translational funding for Scientific \& Technological Achievements, Jilin Science and Technology Development Program.

\section{Authors' contributions}

Jiatong Chai wrote the main manuscript text.

Yiting Wang helped to make Figures.

Zeyu Sun helped Chai screen the publications.

Chun Yang provided the bacteria message.

Jiancheng Xu guided the writing process of the thesis. All authors reviewed the manuscript.

\section{Acknowledgement}

Acknowledgement is given to the participant and her family.

\section{References}

1. Nielsen H, Hansen KK, Gradel KO, Kristensen B, Ejlertsen T, Østergaard C, et al. Bacteraemia as a result of Campylobacter species: a population-based study of epidemiology and clinical risk factors. Clin Microbiol Infect. 2010;16:57-61.

2. do Nascimento Veras H, Medeiros PHQS, Ribeiro SA, Freitas TM, Santos AKS, Amaral MSMG, et al. Campylobacter jejuni virulence genes and immuneinflammatory biomarkers association with growth impairment in children from Northeastern Brazil. Eur J Clin Microbiol Infect Dis. 2018;37:2011-20.

3. Anvarinejad M, Amin Shahidi M, Pouladfar GR, Dehyadegari MA, Mardaneh J. Campylobacter jejuni Bacteremia in a Patient With Acute Lymphocytic Leukemia. Iran Red Crescent Med J [Internet]. 2016 [cited 2021 Jul 23];18. Available from: https://sites.kowsarpub.com/ircmj/articles/55211.html.

4. Yoon JG, Lee SN, Hyun HJ, Choi MJ, Jeon JH, Jung E, et al. Campylobacter jejuni Bacteremia in a Liver Cirrhosis Patient and Review of Literature: A Case Study. Infect Chemother. 2017;49:230.

5. Kaakoush NO, Castaño-Rodríguez N, Mitchell HM, Man SM. Global Epidemiology of Campylobacter Infection. Clin Microbiol Rev. 2015;28:687-720.

6. Pigrau C, Bartolome R, Almirante B, Planes A, Gavalda J, Pahissa A. Bacteremia Due to Campylobacter Species: Clinical Findings and Antimicrobial Susceptibility Patterns. CLIN INFECT DIS. 1997;25:1414-20.

7. Blaser MJ. Editorial, Response. Campylobacter fetus --Emerging Infection and Model System for Bacterial Pathogenesis at Mucosal Surfaces. CLIN INFECT DIS. 1998;27:256-8.

8. Genderini FG, Martiny D, Ponthieux F, Argudín MA, Gomez Galdon M, Zaarour A, et al. First case of Campylobacter rectus and Solobacterium moorei mixed bacteraemia successfully identified by MALDI TOF-MS. New Microbes and New Infections. 2019;31:100587. 
9. Hadano Y, Iwata H. An unusual cause of spontaneous bacterial peritonitis due to Campylobacter fetus with alcoholic liver cirrhosis. Case Reports. 2013;2013:bcr2012008406-6.

10. Singhal N, Kumar M, Kanaujia PK, Virdi JS. MALDI-TOF mass spectrometry: an emerging technology for microbial identification and diagnosis. Front Microbiol [Internet]. 2015 [cited 2021 Jul 23];6. Available from: http://journal.frontiersin.org/Article/10.3389/fmicb.2015.00791/abstract.

11. Fernández-Cruz A, Muñoz P, Mohedano R, Valerio M, Marín M, Alcalá L, et al. Campylobacter Bacteremia: Clinical Characteristics, Incidence, and Outcome Over 23 Years. Medicine. 2010;89:319-30.

12. Pisipati S, Zafar A, Zafar Y. Campylobacter coli bacteraemia: how common is it? BMJ Case Rep. 2020;13:e236634.

13. Kweon OJ, Lim YK, Yoo B, Kim HR, Kim T-H, Lee M-K. First Case Report of Campylobacter volucris Bacteremia in an Immunocompromised Patient. Ledeboer NA, editor. J Clin Microbiol. 2015;53:1976-8.

14. Wang C-H, Tai T-H, Weng S-Y, Yeh S-W, Shiue S-J, Jargalsaikhan G, et al. Spontaneous bacterial peritonitis caused by Campylobacter Coli in cirrhotic patient: A rare case report (CARE-compliant). Medicine. 2020;99:e19887.

15. Gharamti AA, Moukalled N, Taher A, Kanafani ZA. Recurrent Campylobacter Bacteremia as the First Manifestation of Hypogammaglobulinemia: A Case Report and Literature Review. Infect Chemother. 2020;52:415.

\section{Figures}

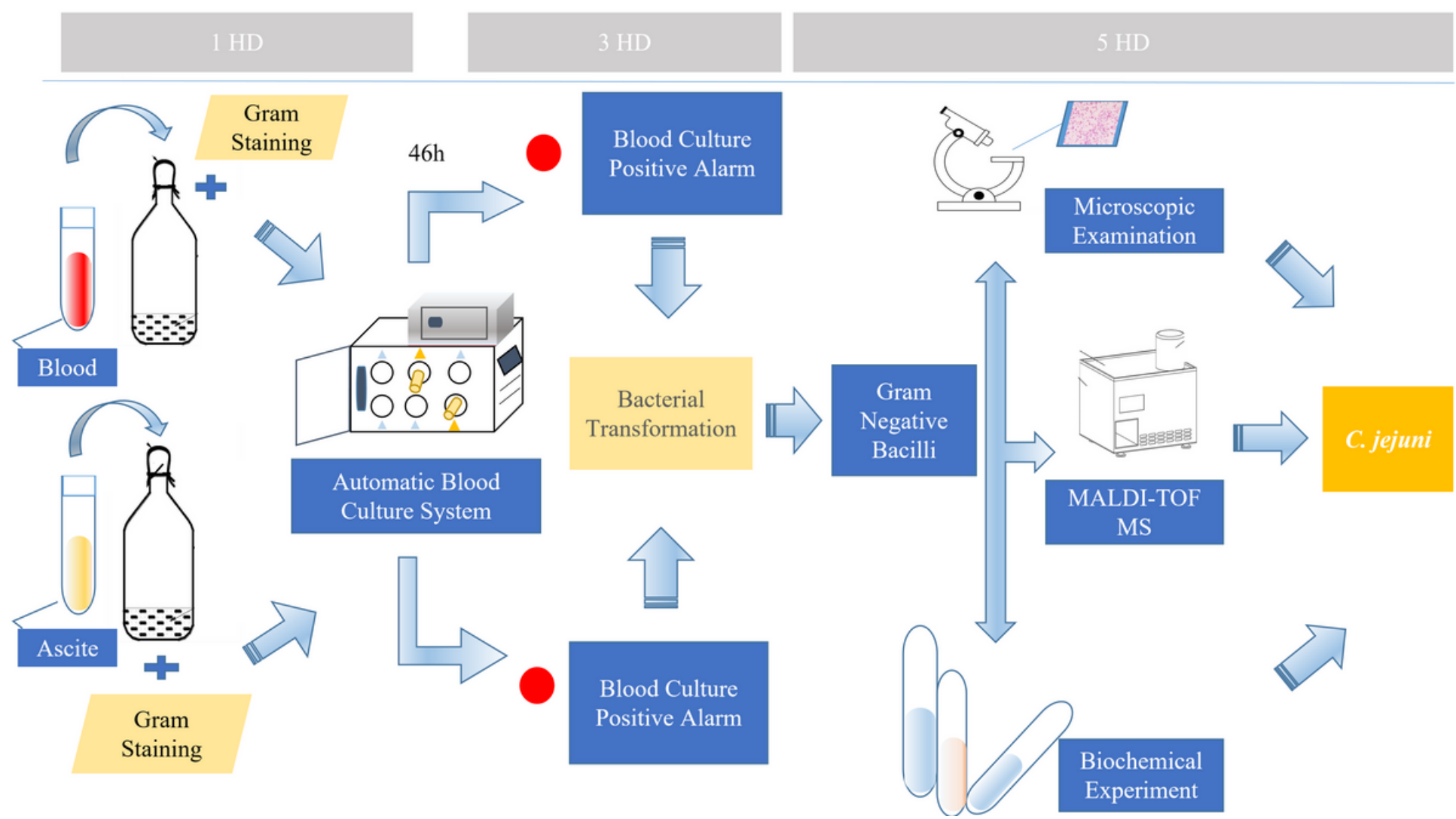

Figure 1

The process of identification. h, hour; HD, hospitalization day. 

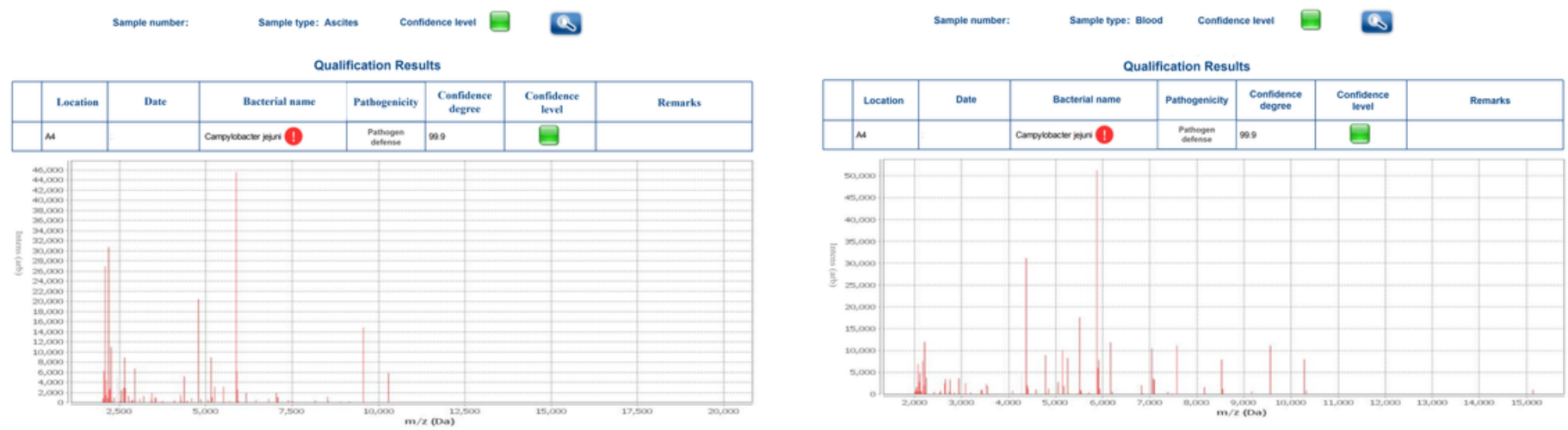

\section{Figure 2}

Pathogen identification results by MALDI-TOF MS. $a$, the result of ascite; $b$, the result of blood. The sample number and test date have been hidden to protect privacy.

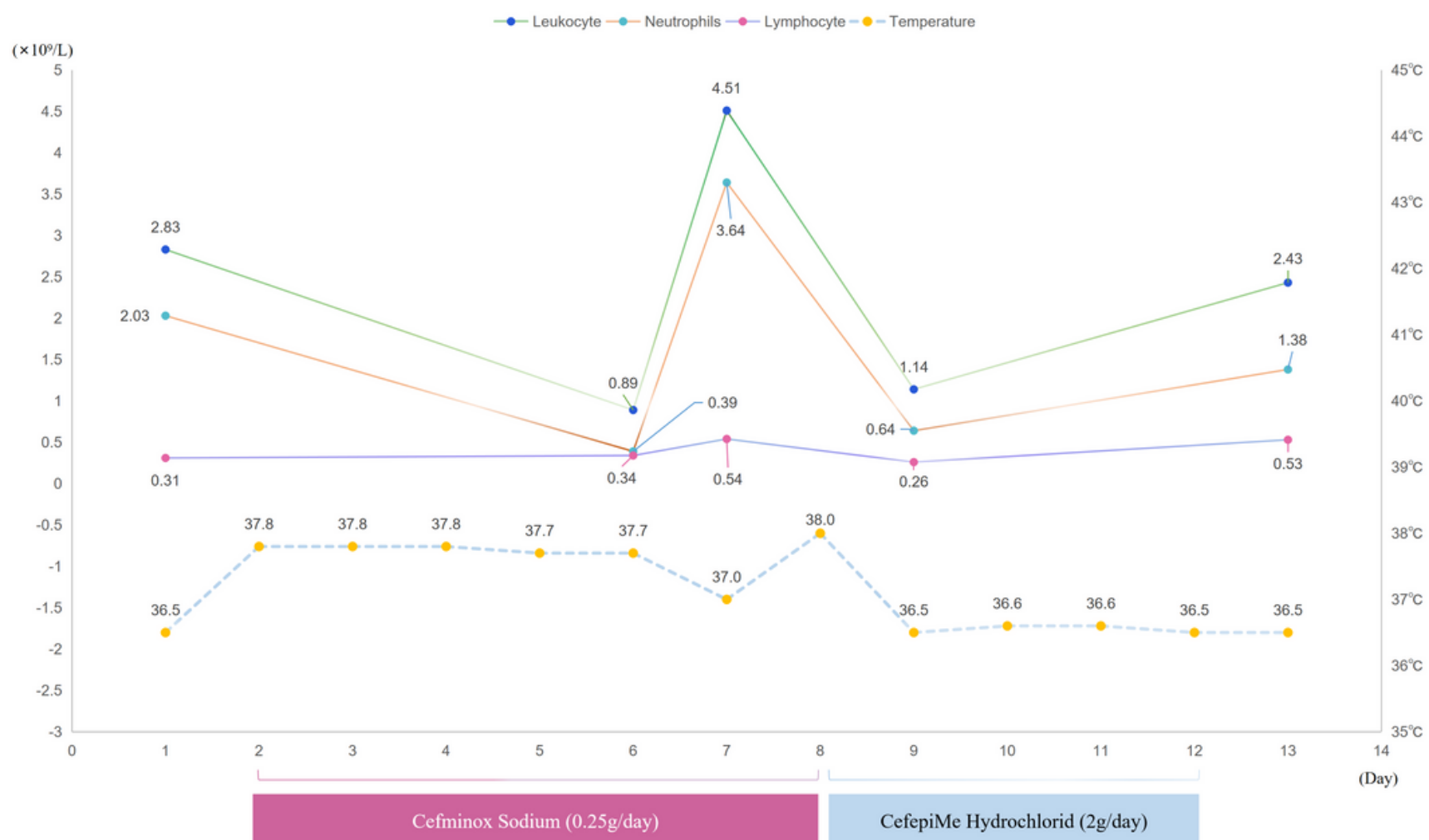

Figure 3

The dynamic changes of test indicators during the hospitalization. The left y-axis represented the levels of leukocytes, neutrophils and lymphocytes, the right $y$-axis represented temperature level. The $x$-axis represented the days of hospitalization. The colored box near the x-axis represents the medication. 


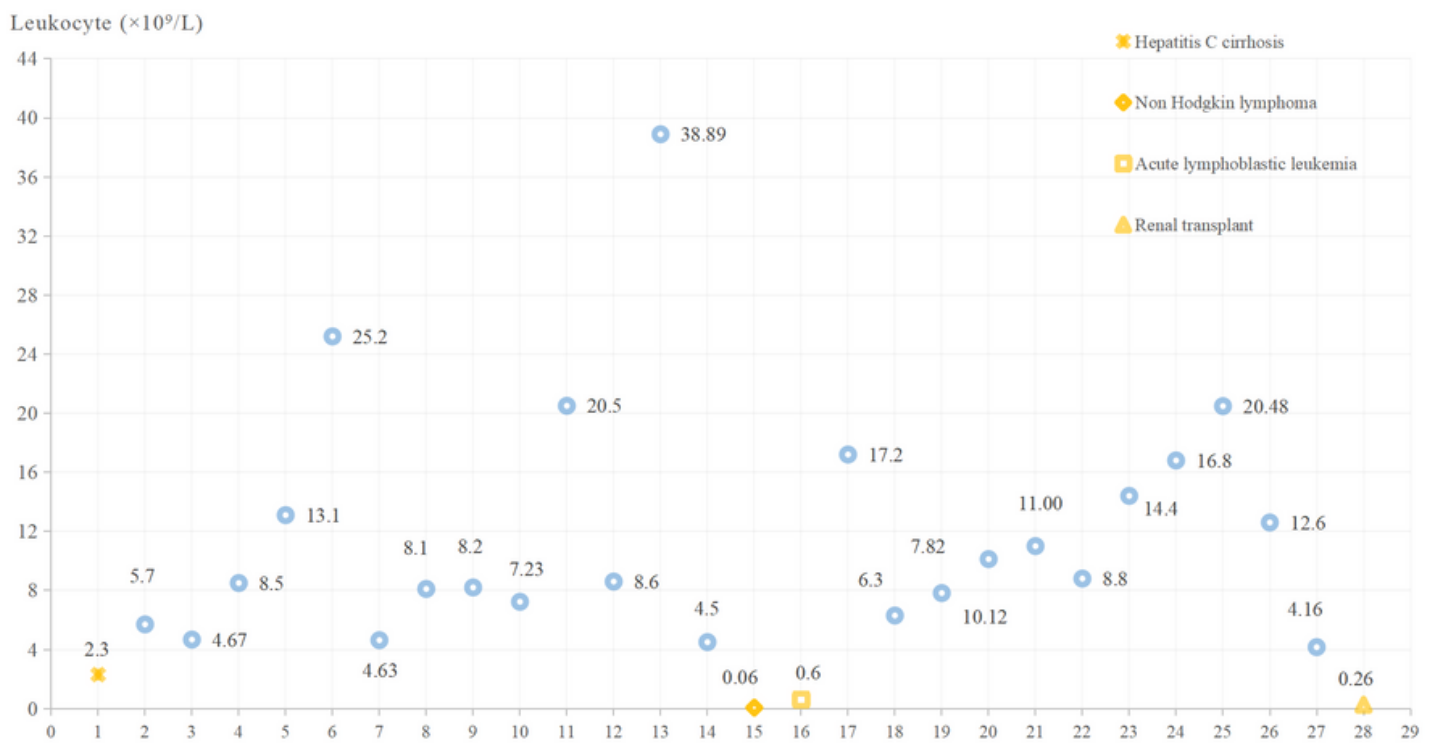

\section{Figure 4}

The levels of leukocyte in previous cases about Campylobacter bacteremia. The abscissa indicates the article number; Each dot represents the leukocyte level of one case, and the number next to the dot is the count of leukocytein in each case. The yellow dot represents the cases with reduced leukocyte level, and the legend on the right represents the comorbidities to the case. 